\title{
Lactobacillus Aggravate Bile Duct Ligation-Induced Liver Inflammation and Fibrosis in Mice
}

\author{
Yoon Seok Roh ${ }^{1}$, Ara Cho ${ }^{2}$, Youn-Soo Cha ${ }^{3}$, Suk-Heung $\mathrm{Oh}^{4}$, Chae Woong Lim ${ }^{2}$ and Bumseok Kim ${ }^{2}$ \\ 'Department of Pharmacy, Chungbuk National University, College of Pharmacy and Medical Research Center, \\ Cheongju, Korea \\ ${ }^{2}$ Biosafety Research Institute and College of Veterinary Medicine (BK21 Plus Program), Chonbuk National University, \\ Iksan, Korea \\ ${ }^{3}$ Department of Food Science and Human Nutrition, Fermented Food Research Center, Chonbuk National University, \\ Jeonju, Korea \\ ${ }^{4}$ Department of Food \& Biotechnology, Woosuk University, Jeonju, Korea
}

\begin{abstract}
Lactobacillus (LAB) have been reported to exert both harmful and beneficial effects on human and animal health. Recently, it has been reported that dysbiosis and bacterial translocation contribute to liver fibrosis. However, the role of Gram-positive LAB in the situation of chronic liver diseases has not been yet elucidated. Liver injury was induced by bile duct ligation (BDL) in LAB or control-administered mice. Liver fibrosis was enhanced in LABadministered mice compared with control-treated mice as demonstrated by quantification of Sirius-red positive area, hydroxyproline contents and fibrosis-related genes (Coll $\alpha 1$, Acta2, Timp1, Tgfb1). Moreover, LAB-administered mice were more susceptible to BDL-induced liver injury as shown by increased ALT and AST level of LAB group compared with control group at 5 days post BDL. Consistent with serum level, inflammatory cytokines (TNF- $\alpha$, IL-6 and IL-1 $\beta$ ) were also significantly increased in LAB-treated mice. Of note, LAB-treated liver showed increased lipoteichoic acid (LTA) expression compared with control-treated liver, indicating that LABderived LTA may translocate from intestine to liver via portal vein. Indeed, responsible receptor or inflammatory factor (PAFR and iNOS) for LTA were upregulated in LAB-administered group. The present findings demonstrate that administration of LAB increases LTA translocation to liver and induces profibrogenic inflammatory milieu, leading to aggravation of liver fibrosis. The current study provides new cautious information of LAB for liver fibrosis patients to prevent the detrimental effect of LAB supplements.
\end{abstract}

Key words: LAB, Liver fibrosis, LTA

\section{INTRODUCTION}

Lactobacillus (LAB) have been used widely in foods and dairy products for over a hundred years. Recently, there has been increasing interest in their use to prevent or treat various diseases. Recently, the Agency for Healthcare

Correspondence to: Bumseok Kim, Laboratory of Pathology, College of Veterinary Medicine, Chonbuk National University, Iksan, Jeonbuk 54596, Korea

E-mail: bskims@jbnu.ac.kr

This is an Open-Access article distributed under the terms of the Creative Commons Attribution Non-Commercial License (http:// creativecommons.org/licenses/by-nc/3.0) which permits unrestricted non-commercial use, distribution, and reproduction in any medium, provided the original work is properly cited.
Research and Quality (AHRQ) concluded that, although the existing $\mathrm{LAB}$ clinical trials reveal no evidence of increased risk, "the current literature is not well equipped to answer questions on the safety of LAB in intervention studies with confidence (1)." Because LAB have been shown to affect both the innate and adaptive immune systems, including effects on cytokine production and immune cell function (2-5), concern has been raised about the potential to overly stimulate the immune response in some individuals, possibly leading to excessive inflammation or autoimmune phenomena. Furthermore, this concern has not been reported in any liver diseases.

Liver fibrosis is a consequence of the chronic wound healing response to continuous hepatocellular damage. Such injury results in a strong inflammatory response 
within the liver, activating hepatic stellate cells to produce large amounts of extracellular matrix (6). Liver cirrhosis, the end stage of liver fibrosis is associated with development of various complications such as ascites, renal failure, hepatic encephalopathy, and portal hypertension, and may progress to hepatocellular carcinoma (7). Thus, investigating the pathogenesis of liver fibrosis is the key step in understanding a number of life-threatening complications in chronic liver disease.

$\mathrm{LAB}$ has been proposed to modulate various inflammatory conditions in body $(8,9)$. Especially, LAB-derived bacterial products, like lipoteichoicacid (LTA), translocate to the liver via portal vein and can mediate several immune responses with participation of inflammatory cytokines. LTA can bind to Toll-like receptor 2 (TLR2) or platelet activating factor receptor (PAFR) present in hepatic cells, which triggers the release of TNF $\alpha$, IL-6, IL1 $\beta$, and other inflammatory cytokines (10). In addition, This signaling promote the production of collagen by activated-HSC (2, 11). Because of the importance of gut-liver axis in fibrosis progression, it has been hypothesized that the change of intestinal microbiota using LAB could modulate the gut barrier and inflammatory and fibrogenic response in liver disease. To examine the our hypothesis, we used murine liver fibrosis model induced by common bile duct ligation (BDL) surgery.

The present study examined the in vivo role of $\mathrm{LAB}$ by assessing liver fibrosis induced by BDL in mice with oral administration of LAB. Our results demonstrated that LAB aggravates liver fibrosis by activation of TLR2 signaling.

\section{MATERIALS AND METHODS}

Strain and culture conditions. Lactobacillus sakei was kindly provided by Dr. Suk-heung Oh, the department of Food and Biotechnology, Woosuk University (Wanju, Korea). These LAB were precultured in Lactobacilli MRS broth from BD Difco TM (CA, USA) at $37^{\circ} \mathrm{C}$ for $24 \mathrm{hr}$. Cultured cells were collected and washed two times with PBS by centrifugation at 3,000 g for $5 \mathrm{~min}$. For oral administration, mice received $10^{9} \mathrm{CFU}$ of LAB resuspended in PBS or PBS only ( $200 \mu \mathrm{L} /$ mouse) for either 5 or 21 consecutive days.

Mice and liver injury model. C57BL/6 mice were maintained in a standard condition $\left(24 \pm 2^{\circ} \mathrm{C}, 50 \pm 5 \%\right.$ humidity), pathogen-free environment and fed a sterile standard rodent chow diet and water ad libitum. Experimental and animal management procedures were undertaken in accordance with the requirements of the Animal Care and Ethics Committees of Chonbuk National University.

For BDL procedures, mice were anesthetized Zoletil (Virbac, $1.6 \mu \mathrm{L} / \mathrm{g}$ ) by intramuscular injection. Sham-operated wt mice, used as controls, underwent laparotomy with exposure but no ligation of the common bile duct. The fascia and skin of the midline abdominal incision were closed with sterile surgical 6-0 sutures (Dafilon; B.Braun/Aesculap AG, Tuttlingen, Germany). C57BL/6 mice underwent BDL. After either 5 days (BDL, $\mathrm{n}=8$ per group; Sham, $\mathrm{n}=4$ per group) or 21 days (BDL, $n=8$ per group; Sham, $n=4$ per group) of surgery, mice were reanesthetized, and blood was obtained directly from the heart for serum hepatic enzyme determinations.

Determination of liver fibrosis by Sirius red staining. Liver fibrosis was quantified at 21 days post BDL using Sirius red staining as described by Kim et al (12). Direct red 80 was obtained from Sigma-Aldrich Diagnostics. Liver sections of were stained, and red-stained collagen fibers were quantified by the percentage of positive area per total liver section. Total liver section images were analyzed in individual animals using a light microscope (BX51, Olympus Corp., Tokyo, Japan) and digital image software (analySIS TS, Olympus Corp., Tokyo, Japan). Data were expressed as the percentage of Sirius red-positive area per field.

Measurement of hepatic hydroxyproline content. Hydroxyproline content was measured as previously described (13). Liver specimens were weighed and $20 \mathrm{mg}$ of freeze-dried sample was hydrolyzed in $6 \mathrm{~N} \mathrm{HCl}$ at $120^{\circ} \mathrm{C}$ for $12 \mathrm{hr}$. Fifty $\mu \mathrm{L}$ of each hydrolyzed sample was transferred to a 96-well plate and evaporated to dryness under vacuum. The hydrolysate was evaporated under vacuum and the sediment was re-dissolved in $1 \mathrm{~mL}$ distilled water. Samples were incubated with chloramine-T solution for $20 \mathrm{~min}$ at room temperature, followed by incubation in Ehrlich's solution at $65^{\circ} \mathrm{C}$ for $20 \mathrm{~min}$. After cooling, the absorbance was read at $561 \mathrm{~nm}$. Hydroxyproline concentration was calculated from a standard curve prepared with high purity hydroxyproline. The results were expressed as $\mu \mathrm{g}$ hydroxyproline per $10 \mathrm{mg}$ of liver protein.

Quantitative real-time polymerase chain reaction ( $\boldsymbol{q} P \boldsymbol{C} \boldsymbol{R})$. We extracted total RNA from liver tissue using the Easy-Spin Total RNA extraction kit (iNtRon Biotech, Seoul, Korea). Following incubation with RNase-free DNase I (Promega, Madison, WI, USA), reverse transcription was performed using the High Capacity cDNA Reverse Transcription Kit (Applied Biosystems, Foster City, CA, USA) according to the manufacturer's instructions. cDNA was subjected to real-time PCR on a CFX96 ${ }^{\mathrm{TM}}$ Real-Time PCR Detection System (Bio-Rad Laboratories, CA, USA) using SYBR Green I as a double-strand DNA-specific binding dye. After the reaction was completed, we verified specificity by melting curve analysis. Quantification was performed by comparing $\mathrm{Ct}$ values of each sample after normalization to GAPDH. Sequences of primers were 
summarized in Supplementary Table 1.

Serum liver enzyme quantification. We measured serum levels of alanine aminotransferase (ALT), aspartate aminotransferase (AST), and alkaline phosphate (ALP) using the Roche Diagnostics COBAS INTEGRA 400 Plus (Indianapolis, IN, USA).

Immunoblot analysis. Liver tissue was directly lysed with an extraction buffer (T-PER, Thermo Fisher Scientific Inc., Rockford, IL, USA) for 30 min on ice. After centrifugation at $13,000 \mathrm{~g}$ for $15 \mathrm{~min}$ at $4^{\circ} \mathrm{C}$, protein concentration in the supernatant was measured using Bradford's reagent (Thermo Fisher Scientific Inc.). Protein $(30 \mu \mathrm{g})$ was resolved by sodium dodecyl sulfate-polyacrylamide gel electrophoresis (SDS-PAGE) on a gradient gel and then transferred onto PVDF membranes. Blocking was carried out using blocking buffer $[5 \%$ nonfat dairy milk in Tris-buffered saline $(20 \mathrm{mM}$ Tris, $150 \mathrm{mM} \mathrm{NaCl}, \mathrm{pH}$ 7.4) containing $0.05 \%$ Tween-20] for $1 \mathrm{hr}$ at room temperature. Primary antibodies were diluted 1:1,000 in a blocking buffer and incubated at $4{ }^{\circ} \mathrm{C}$ overnight. The following antibodies were used: anti-LTA (mouse antibody, Invitrogen, Waltham, MA, USA). To detect antigen antibody complexes, antirabbit or anti-mouse horseradish peroxidase (HRP)-conjugated secondary antibodies (Santa Cruz Biotechnology Inc., Santa Cruz, TX, USA) were diluted 1:3000 in blocking buffer and incubated at room temperature for $45 \mathrm{~min}$. Immune complexes were visualized using chemiluminescent substrate (Millipore, Burlington, MA, USA) according to the manufacturer's instructions.

Statistical analysis. All data were expressed as mean \pm standard error. Differences between two groups were compared using a two-tailed Student's $t$-test. A value of $p<0.05$ was considered statistically significant.
(A)
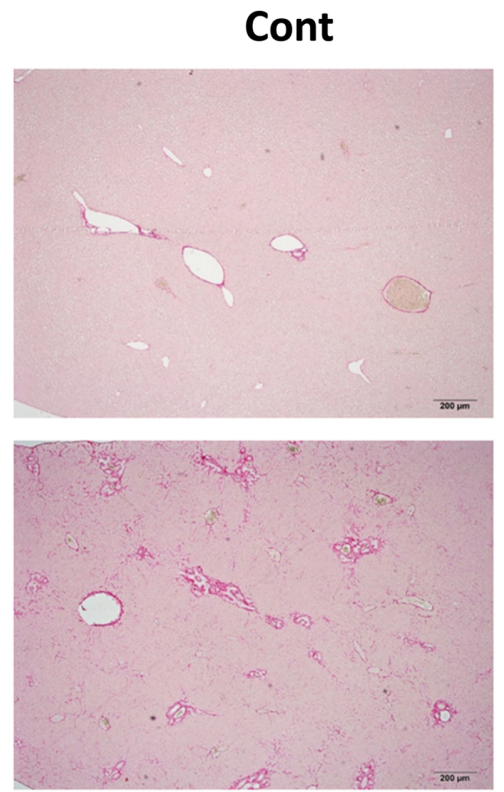

(B)

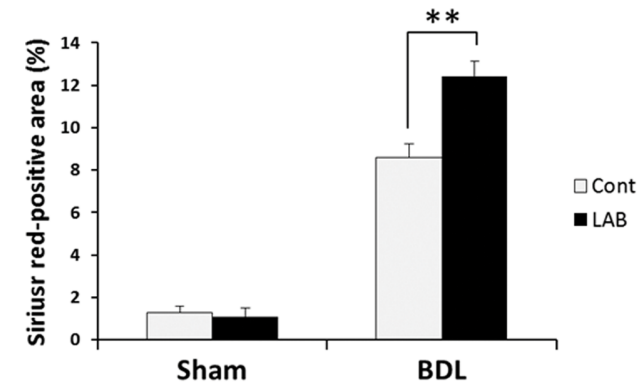

LAB
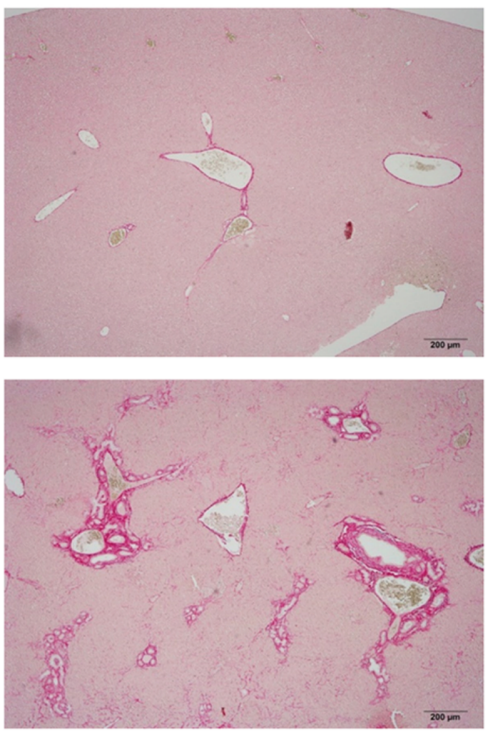

BDL

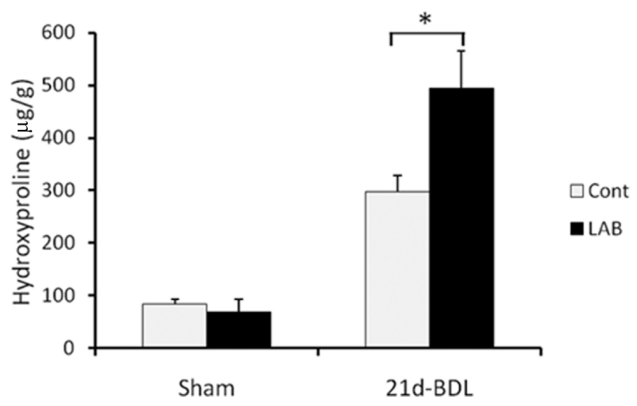

Fig. 1. Administration of $L A B$ exacerbates $B D L$-induced liver fibrosis. (A-B) Control or $L A B$-administered mice underwent sham operation ( $n=4$ per group) or BDL for 21 days ( $n=8$ per group). Fibrillar collagen deposition was determined by quantification of the Sirius red-positive area and hydroxyproline contents. LAB administration induced significant increase of Sirius red-positive area and hydroxyproline contents compared with control group. Data are presented as means \pm SEM per group. Two-tailed Student's $t$ test, ${ }^{*} p<0.05,{ }^{* *} p<0.01$. Original magnification, $\times 200$ (Sirius-Red). 


\section{RESULTS}

\section{Administration of $L A B$ exacerbates $B D L$-induced liver}

fibrosis. To investigate the effect of LAB in liver fibrosis, LAB or control vehicle-treated WT mice were subjected to BDL. At 21 days after BDL, LAB-administered mice displayed significantly increased liver fibrosis compared with control-treated mice as determined by quantification of Sirius red-positive area, which is specific for collagen deposition. Furthermore, hydroxyproline content was also significantly increased in LAB-administered mice (Fig. 1A, 1B). Therefore, these results suggest that
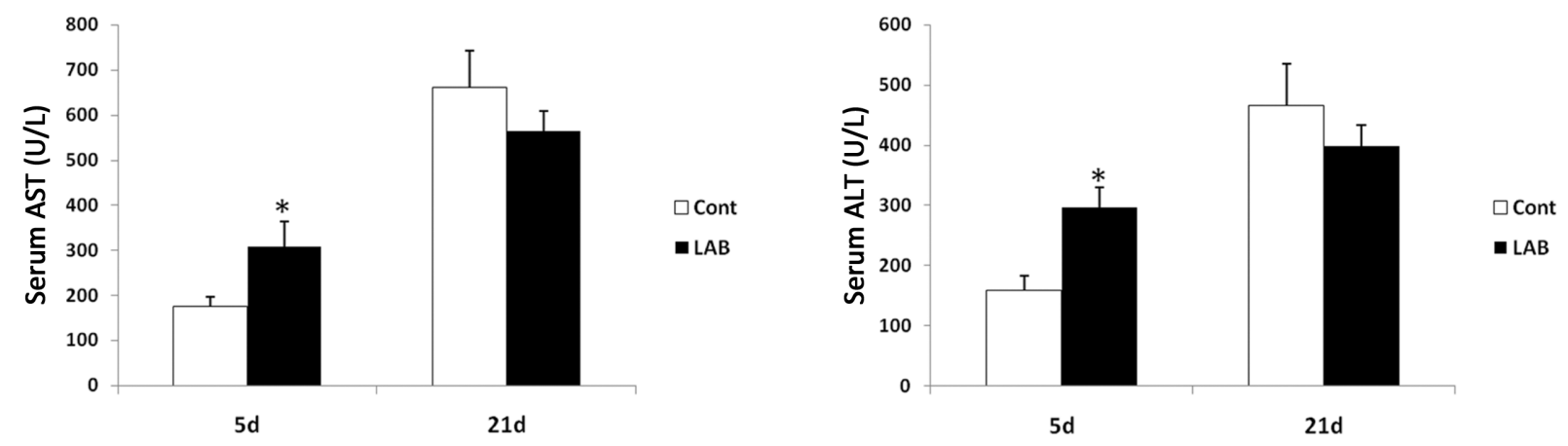

Fig. 2. LAB-administered mice are more susceptible to BDL-induced liver injury. (A) ALT level of LAB group were significantly higher than control group at 5 days post BDL. Data are presented as means \pm SEM per group. Two-tailed Student's $t$-test, ${ }^{*} p<0.05$.

(A)
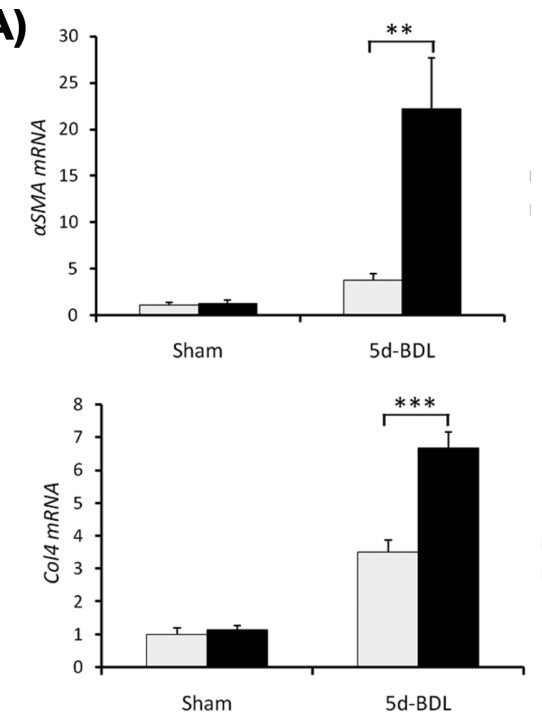

(B)

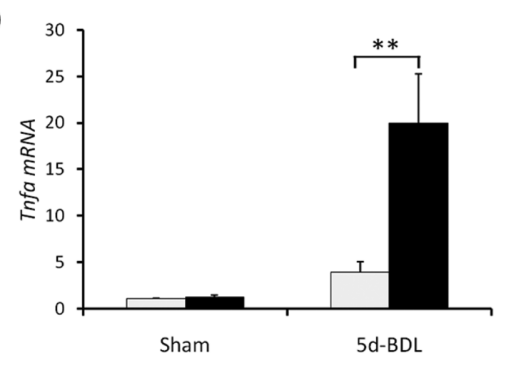

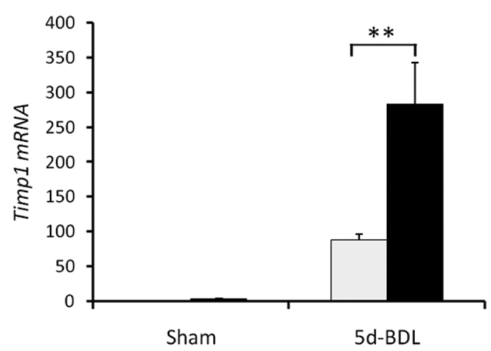
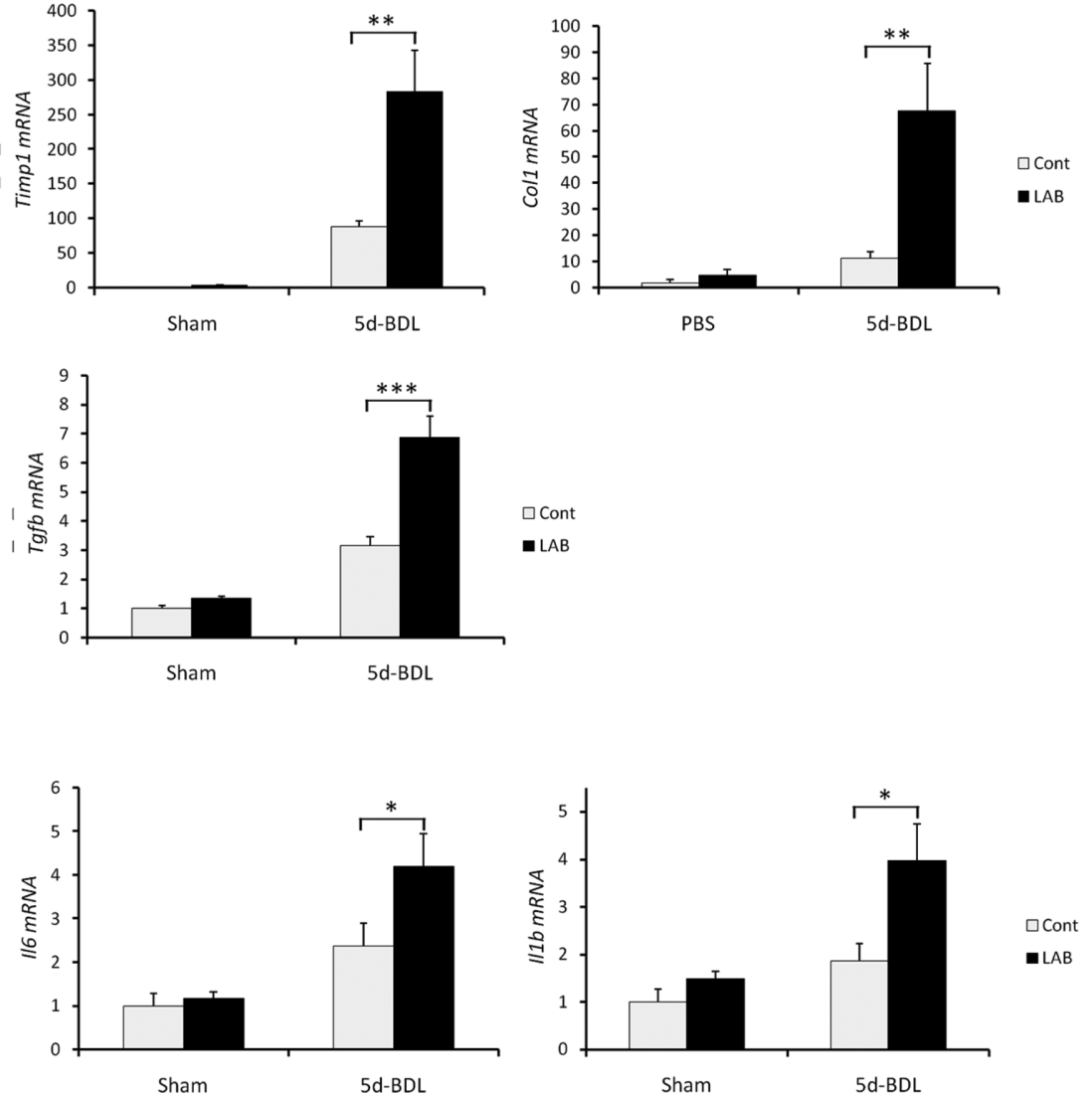

Fig. 3. $L A B$ administration favors progression of liver fibrosis. (A-B) Control or LAB-administered mice underwent sham operation ( $n=4$ per group) or BDL for 5 days ( $n=6$ per group). Expression of genes were determined by quantitative real time PCR. (A) Fibrosis related genes in liver were increased in LAB-administered group compared with control-treated group. (B) Various proinflammatory genes were also significantly increased in LAB-treated group. Data are presented as means \pm SEM per group. Two-tailed Student's $t$-test, ${ }^{*} p<0.05,{ }^{* *} p<0.01,{ }^{* * *} p<0.001$. 
LAB administration enhances liver fibrosis induced by BDL.

LAB-administered mice are more susceptible to BDLinduced liver injury. Next, we investigated the effect of LAB on liver injury induced by BDL. Consistent with results of Fig. 1, increased liver injury in LAB-treated group was confirmed by increased serum ALT and AST levels (Fig. 2) at 5 days after BDL. Thus, LAB may increase the susceptibility of cholestasis-induced liver injury.

LAB administration favors progression of liver fibrosis. The exacerbation of liver fibrosis was also confirmed by qRT-PCR analysis of hepatic expression of fibrogenic genes (Fig. 3). The mRNA levels of Colla1, Col4, Acta2, Timp1, and Tgfb1 were significantly elevated in LABtreated group compared with control group (Fig. 3A). Furthermore, inflammatory responses of LAB-administered mice were significantly higher than those of controltreated mice as demonstrated by hepatic expression of proinflammatory cytokines including IL- $1 \beta$, TNF- $\alpha$ and IL-6 (Fig. 3B). These results suggest that LAB promotes profibrogenic milieu of cholestasis of liver injury.

LAB-treated mice show increased hepatic LTA expression and related responses. Above results (Fig. 1-3) prompted us to test whether oral administration LAB affects the translocation of lactobacillus LTA to liver. Indeed, LAB-treated liver showed increased LTA expression compared with control-treated liver, indicating that LAB-originated LTA may translocate from intestine to liver via portal vein (Fig. 4A). Moreover, LTA-related receptor or inflammatory factor (PAFR and iNOS) were significantly upregulated in LAB-administered group compared with control group. Collectively, these results indicate that oral administration of LAB increases the LTA translocation to liver and inflammatory signaling.

\section{DISCUSSION}

We demonstrate herein that $\mathrm{LAB}$ administration exacerbates liver fibrosis in mice. LAB increased the expressions of profibrogenic and proinflammatory cytokines and exhibited increased the susceptibility to BDL-induced liver injury. Because LAB-originated LTA could translocates to activate the hepatic inflammation, their administration leads to increased inflammation and progression of liver fibrosis.

Previously, many studies have been investigated the beneficial effects of LAB on various diseases. However, little is known about the side effect of exposure to $\mathrm{LAB}$ in human and animals. Specifically, it has been reported that LAB are closely related with inflammation of gut-liver axis $(9,14)$. Therefore, we used the BDL-induced liver fibrosis model to study LAB-mediated inflammation and subsequent liver fibrosis.

Unexpectedly, we found that LAB aggravated liver injury and fibrosis. The translocation of intestinal bacteria to liver have been suggested to be a key step in the pathogenesis of liver fibrosis (14). In current study, LAB-originated LTA was translocated to liver. It has been reported that LTA from gram-positive bacteria induces nitric oxide production using a PAFR signaling pathway to activate STAT1 via Jak2 (15). Consistently, our data showed LAB

(A)

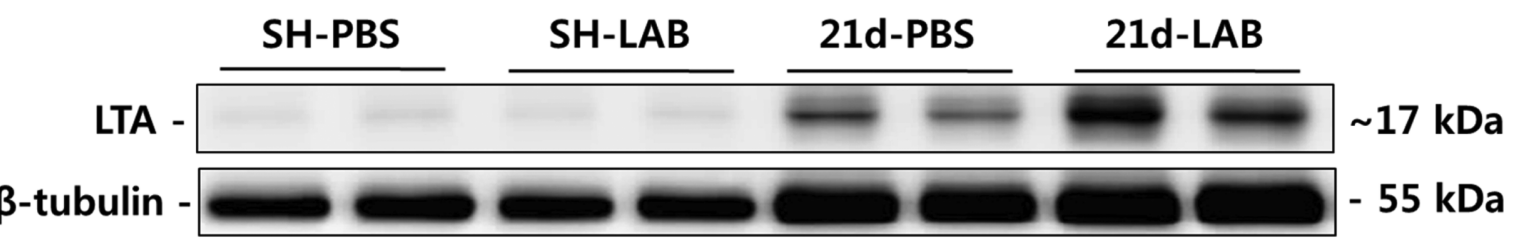

(B)
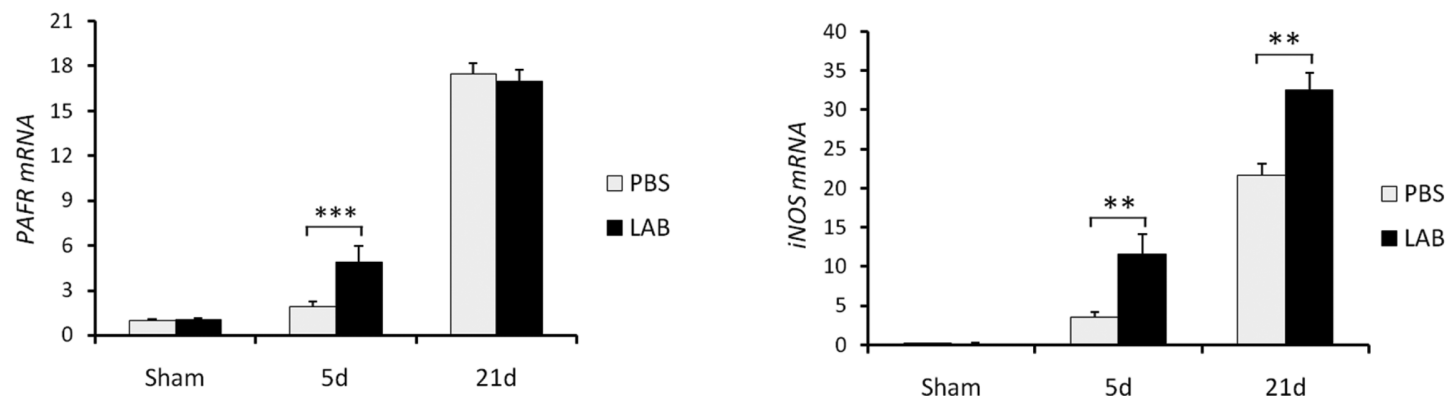

Fig. 4. $L A B-t r e a t e d$ mice shows increased hepatic LTA expression and related responses. (A-B) Hepatic expression of LTA were measured by western blot. (A) LTA expression in LAB group were much higher than control group. (B) LTA-related receptor or inflammatory factor (PAFR and iNOS) were also upregulated in LAB-administered group. Two-tailed Student's $t$-test, ${ }^{* *} p<0.01,{ }^{* * *} p<0.001$. 
increased the expression of PAFR and NO. Therefore, LAB could be a detrimental factor for liver disease patients through excessive stimulation of liver immune system.

The exact mechanisms responsible for exacerbation of liver fibrosis in LAB-administered mice remain unclear, but the following data are relevant. First, LAB-treated mice generated high levels of inflammatory cytokines (TNF- $\alpha$, IL-6 and IL-1 $\beta$ ) and nitric oxide, which are potent activators of the immune system in the liver (16). Second, we showed that administration of LAB enhances susceptibility to liver injury (ALT and AST), which is critical event in the course of liver fibrosis (17). Third, we observed that livers of LAB treated mice have increased translocation of bacterial LTA, which is believed to directly promote fibrosis (18). Accumulating evidence suggests that LTA is a ligand for PAFR as well as TLR2 signaling. Furthermore these receptors have been reported to express on hepatic stellate cells (HSCs) and Kupffer cells (KCs), which are responsible cell type for liver fibrognenesis $(11,19)$.

In conclusion, $\mathrm{LAB}$ induce the profibrogenic milieu in response to BDL via induction of LTA translocation to liver and may stimulate the PAFR-mediated signaling in order to activate HSCs and KCs in liver fibrosis. Thus, the present study provides additional information of detrimental effect about LAB to pave the way for the development and evaluation of dietary constituents as health supplemental agents.

\section{ACKNOWLEDGMENTS}

This research was supported by a grant (NRF2017R1D1A3B03030521) of the Basic Science Research Program through the National Research Foundation (NRF) funded by the Ministry of Education, Republic of Korea. It was also supported by the grant (NRF2017R1C1B2004423) funded by the Ministry of Science and ICT, Republic of Korea, and by the research grant of the Chungbuk National University in 2016.

\section{CONFLICT OF INTEREST}

None of the authors have any conflicts of interest to disclose.

Received December 18, 2017; Revised March 8, 2018; Accepted May 31, 2018

\section{REFERENCES}

1. Hempel, S., Newberry, S., Ruelaz, A., Wang, Z., Miles, J.N., Suttorp, M.J., Johnsen, B., Shanman, R., Slusser, W., Fu, N., Smith, A., Roth, B., Polak, J., Motala, A., Perry, T. and She- kelle, P.G. (2011) Safety of probiotics used to reduce risk and prevent or treat disease. Evid. Rep. Technol. Assess. (Full Rep.), 200, 1-645.

2. Vaarala, O. (2003) Immunological effects of probiotics with special reference to lactobacilli. Clin. Exp. Allergy, 33, 16341640.

3. Veckman, V., Miettinen, M., Pirhonen, J., Siren, J., Matikainen, S. and Julkunen, I. (2004) Streptococcus pyogenes and Lactobacillus rhamnosus differentially induce maturation and production of Th1-type cytokines and chemokines in human monocyte-derived dendritic cells. J. Leukoc. Biol., 75, 764-771.

4. Braat, H., de Jong, E.C., van den Brande, J.M., Kapsenberg, M.L., Peppelenbosch, M.P., van Tol, E.A. and van Deventer, S.J. (2004) Dichotomy between Lactobacillus rhamnosus and Klebsiella pneumoniae on dendritic cell phenotype and function. J. Mol. Med. (Berl.), 82, 197-205.

5. Drakes, M., Blanchard, T. and Czinn, S. (2004) Bacterial probiotic modulation of dendritic cells. Infect. Immun., 72, 3299-3309.

6. Friedman, S.L. (2003) Liver fibrosis -- from bench to bedside. J. Hepatol., 38 Suppl 1, S38-S53.

7. Bataller, R. and Brenner, D.A. (2005) Liver fibrosis. J. Clin. Invest., 115, 209-218.

8. Kang, S.S., Ryu, Y.H., Baik, J.E., Yun, C.H., Lee, K., Chung, D.K. and Han, S.H. (2011) Lipoteichoic acid from Lactobacillus plantarum induces nitric oxide production in the presence of interferon-gamma in murine macrophages. Mol. Immunol., 48, 2170-2177.

9. Vizoso Pinto, M.G., Rodriguez Gomez, M., Seifert, S., Watzl, B., Holzapfel, W.H. and Franz, C.M. (2009) Lactobacilli stimulate the innate immune response and modulate the TLR expression of HT29 intestinal epithelial cells in vitro. Int. J. Food Microbiol., 133, 86-93.

10. Chao, W., Liu, H.L., Zhou, W.G., Hanahan, D.J. and Olson, M.S. (1990) Regulation of platelet-activating factor receptor and platelet-activating factor receptor-mediated biological responses by cAMP in rat Kupffer cells. J. Biol. Chem., 265, 17576-17583.

11. Chen, Y., Wang, C.P., Lu, Y.Y., Zhou, L., Su, S.H., Jia, H.J., Feng, Y.Y. and Yang, Y.P. (2008) Hepatic stellate cells may be potential effectors of platelet activating factor induced portal hypertension. World J. Gastroenterol., 14, 218-223.

12. Kim, J.W., Yun, H., Choi, S.J., Lee, S.H., Park, S., Lim, C.W., Lee, K. and Kim, B. (2017) Evaluating the influence of side stream cigarette smoke at an early stage of non-alcoholic steatohepatitis progression in mice. Toxicol. Res., 33, 31-41.

13. Jung, Y.R., Lee, Y.J., Lee, N.J., Lin, C.M., Moon, J.H., Chai, H.Y. and Kang, J.K. (2010) Inhibitory effect of 1-O-hexyl2,3,5-trimethylhydroquinone on dimethylnitrosamine-induced liver fibrosis in male SD rats. Toxicol. Res., 26, 193-201.

14. Fouts, D.E., Torralba, M., Nelson, K.E., Brenner, D.A. and Schnabl, B. (2012) Bacterial translocation and changes in the intestinal microbiome in mouse models of liver disease. J. Hepatol., 56, 1283-1292.

15. Han, S.H., Kim, J.H., Seo, H.S., Martin, M.H., Chung, G.H., Michalek, S.M. and Nahm, M.H. (2006) Lipoteichoic acidinduced nitric oxide production depends on the activation of 
platelet-activating factor receptor and Jak2. J. Immunol., 176, 573-579.

16. Connolly, M.K., Bedrosian, A.S., Mallen-St Clair, J., Mitchell, A.P., Ibrahim, J., Stroud, A., Pachter, H.L., Bar-Sagi, D., Frey, A.B. and Miller, G. (2009) In liver fibrosis, dendritic cells govern hepatic inflammation in mice via TNF-alpha. $J$. Clin. Invest., 119, 3213-3225.

17. Xu, R., Zhang, Z. and Wang, F.S. (2012) Liver fibrosis: mechanisms of immune-mediated liver injury. Cell. Mol.
Immunol., 9, 296-301.

18. Hartmann, P., Haimerl, M., Mazagova, M., Brenner, D.A., Schnabl, B. (2012) Toll-like receptor 2-mediated intestinal injury and enteric tumor necrosis factor receptor I contribute to liver fibrosis in mice. Gastroenterology, 143, 13301340.e1.

19. Mencin, A., Kluwe, J. and Schwabe, R.F. (2009) Toll-like receptors as targets in chronic liver diseases. Gut, 58, 704720. 